\title{
Recurrence of proptosis caused by a second orbital tumour of different pathology
}

\author{
ALY MORTADA \\ Department of Ophthalmology, Faculty of Medicine, Cairo University
}

The two following cases are described because, some years after the successful surgical removal of a simple encapsulated orbital tumour, proptosis of the same eye recurred because of a second tumour of different pathology. Such a sequence of events has rarely been reported in the literature.

\section{Case reports}

There was no significant family history and no history of trauma or of $x$ ray therapy. There were no signs of endocrine disturbances, and no enlargement of the thyroid gland, liver, spleen, or lymph glands. The chest and abdomen revealed no abnormality. The skin did not show café-au-lait areas of pigmentation, nodules, or haemangiomata. The temperature, pulse, blood pressure, urine, faeces, blood counts, and bleeding and clotting times were normal. The blood Wassermann reaction, tuberculin test, and Casoni's test for hydatid were negative. The basal metabolic rate, and the 131 iodine uptake were normal.

Case r, a 50-year-old man, complained of left proptosis of 5 years' duration (Fig. I).

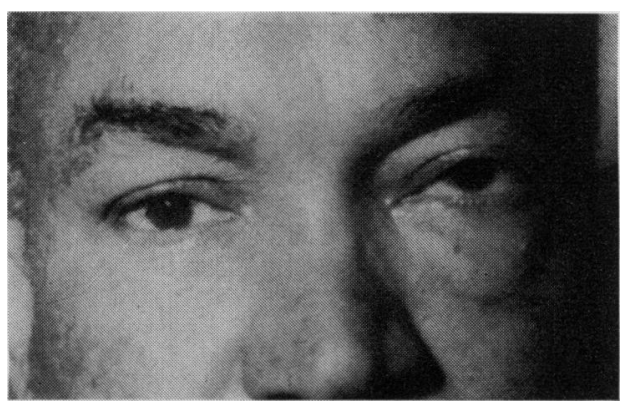

FIG. I Ciase I. Left proptosis due to benign haemangioendothelioma in a man aged 50 years.

\section{EXAMINATION}

The right eye was normal with a normal fundus and the visual acuity was 6/9. The left eye showed proptosis forwards and upwards of $22 \mathrm{~mm}$. Hertel (right side $16 \mathrm{~mm}$.) and limitation of ocular movements downwards. The fundus was normal, and the visual acuity $6 / 18$ with no error of refraction. A soft mass was felt between the lower orbital margin and the eye. Skull $x$ ray showed no change in the orbits or paranasal sinuses.

TREATMENT

A lower fornix conjunctival incision was made and the tumour was located below and towards the 
inner side of the eye. It was not attached to the optic nerve and was removed easily by blunt littlefinger dissection. The tumour was encapsulated and measured $4 \times 2 \mathrm{~cm}$. (Fig. 2); it was soft, nodular, and pinkish grey in colour.

(2)

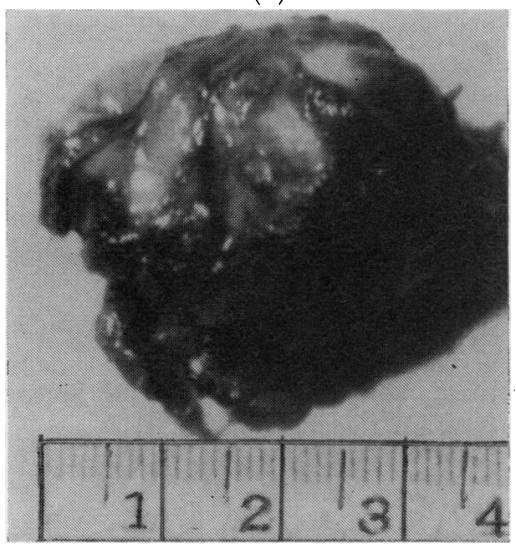

FIG. 2 Case I. Tumour measuring $4 \times 2 \mathrm{~cm}$., pink and encapsulated

FIG. 3 Case I. Section of haemangioendothelioma, showing proliferating endothelial cells surrounding clefts and blood spaces. $\times 540$

FIG. 4 Case I. Section of haemangioendothelioma, showing endothelial cell proliferation inside neoplastic capillaries. Wilder reticulin stain. $\times 540$

(3)
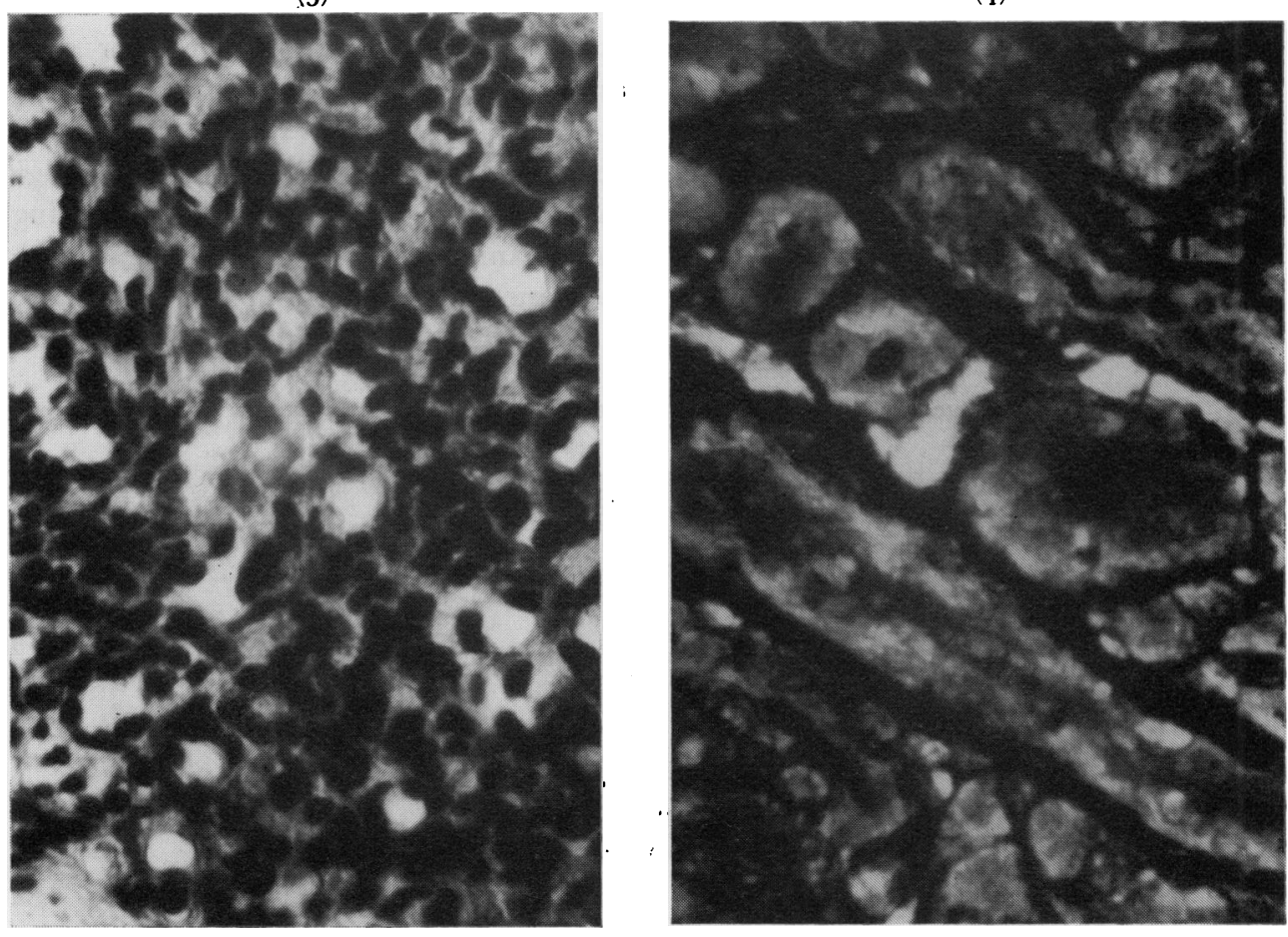

(4)

HISTOPATHOLOGICAL EXAMINATION

It was an encapsulated tumour with a concentric arrangement of proliferating endothelial cells mostly around clefts, blood spaces, and neoplastic capillary vessels (Fig. 3). Wilder reticulin stain showed endothelial cell proliferation inside the capillary lumina (Fig. 4).

DIAGNOSIS

Haemangioendothelioma. 
PROGRESS

The patient continued in perfect health and with no proptosis for 3 years (Fig. 5).

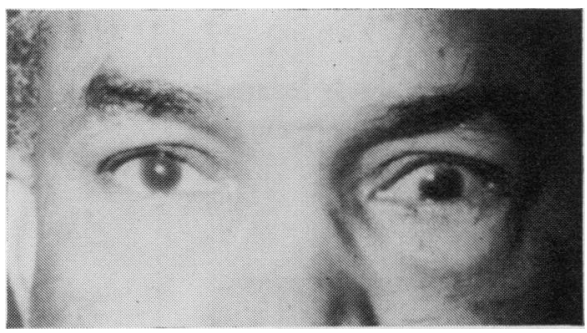

F IG. 5 Case I. 3 years after removal of benign haemangioendothelioma, showing absence of proptosis

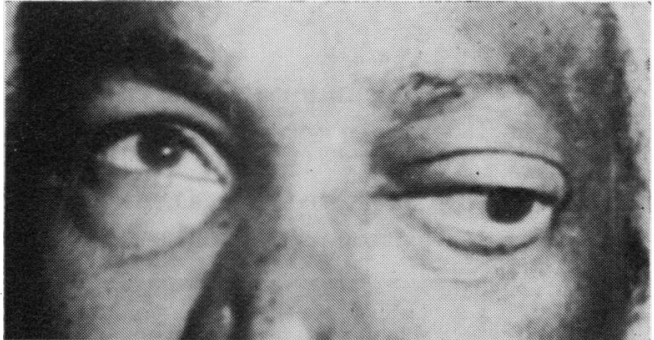

FIG. 6 Case I. After a further 3 years, there was recurrence of the left proptosis, but this time the cause was a maxillary carcinoma.

RE GURRENCE

After another 3 years there was a recurrence of left proptosis (Fig. 6). The right eye remained normal, and the visual acuity was 6/9. The left eye, which was blind, showed proptosis outwards of $26 \mathrm{~mm}$. (Hertel) with some limitation of ocular movements in all directions. The fundus showed optic atrophy. Skull $x$ rays showed some opacity of the left maxillary sinus with destruction of the bony floor of the orbit (Fig. 7). Orbital exploration revealed the extension to the orbit of a maxillary sinus tumour. Histopathological examination of a biopsy of the tumour showed a moderately differentiated squamous cell carcinoma (Fig. 8). Radiotherapy was given but after 2 years the patient died of pneumonia.

(8)
(7)

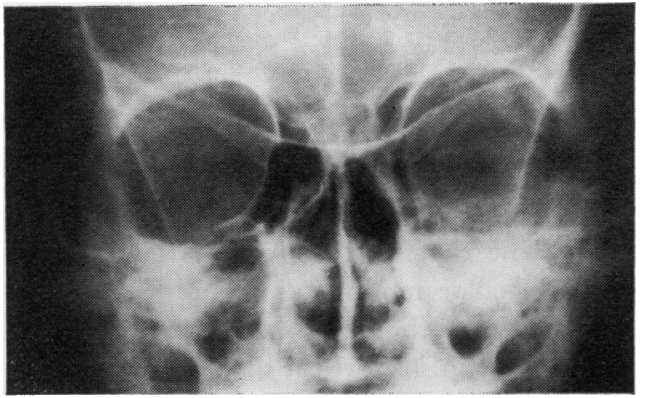

Fig. 7 Case I. Postero-anterior $x$-ray of skull, showing opaque left maxillary antrum and destruction of the orbital bony floor by a carcinoma of the maxillary sinus
FIG. 8 Case I. Section of squamous cell carcinoma of left maxillary antrum invading the orbit. $\times 540$

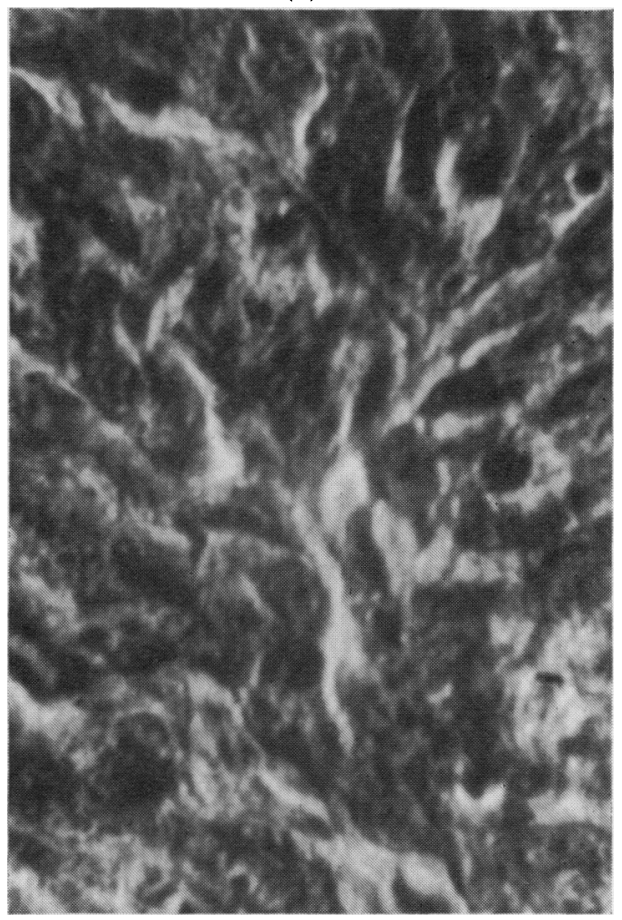

Case 2, a 45-year-old man, complained of right proptosis of 6 years' duration.

EXAMINATION

The left eye was normal with a normal fundus and a visual acuity of $6 / 6$. The right eye showed 
proptosis outwards of $20 \mathrm{~mm}$. Hertel (left side $16 \mathrm{~mm}$.) and slight limitation of ocular movement inwards. The fundus was normal, and the visual acuity 6/12. A soft mass was felt between the inner orbital margin and the eye.

TREATMENT

Through an inner fornix conjunctival incision, the tumour was located at the inner side of the eye. It was soft, encapsulated, and red in colour and measured $3 \times 2 \mathrm{~cm}$.

HISTOPATHOLOGICAL EXAMINATION

It was an encapsulated cavernous haemangioma (Fig. 9).

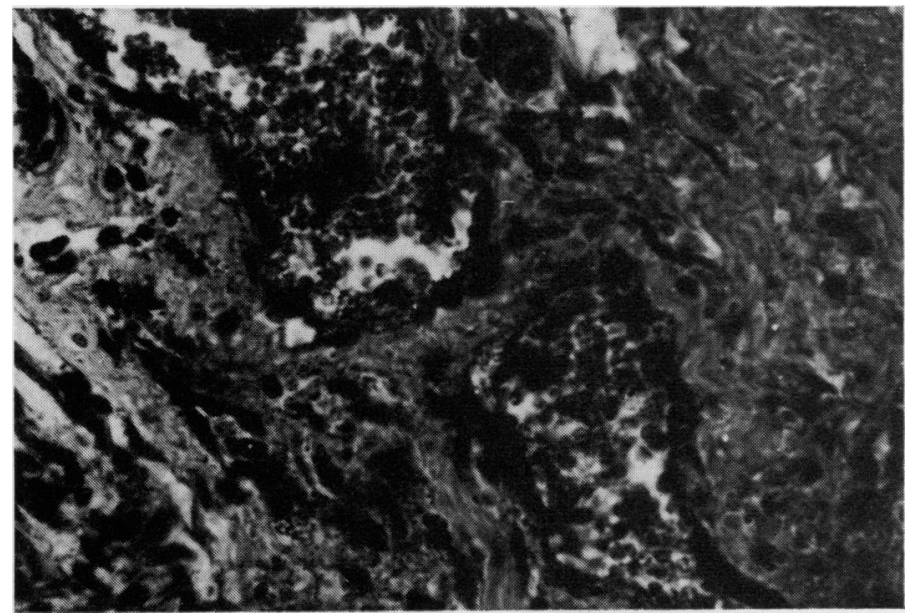

FIG. 9 Case. 2 Section of cavernous haemangioma removed from right orbit of a man aged 45 years. $\times 540$

PROGRESS

The patient remained free of proptosis for 4 years.

RECURRENCE

After another 2 years, however, there was a recurrence of the right proptosis (Fig. 10). The left eye was normal, and the visual acuity 6/6. The right eye, which was blind, showed proptosis upwards of $22 \mathrm{~mm}$. Hertel with limitation of ocular movements in all directions. The fundus showed optic atrophy. Skull $x$ rays showed opacity of the right maxillary sinus with destruction of the bony floor of the right orbit, which was wider in its vertical diameter (Fig. II). The blood

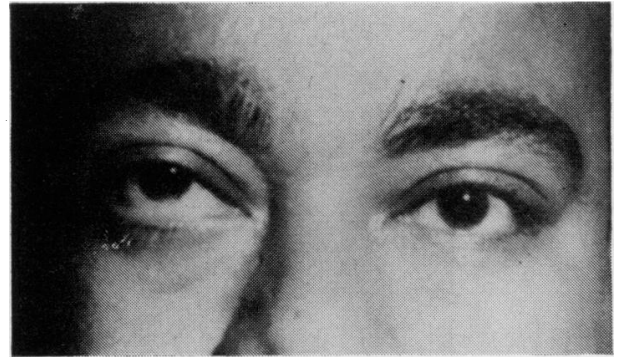

FIG. Io Case 2. 6 years after removal of right orbital cavernous haemangioma, there was recurrence of right proptosis which was due to a right maxillary sinus reticulum cell carcinoma

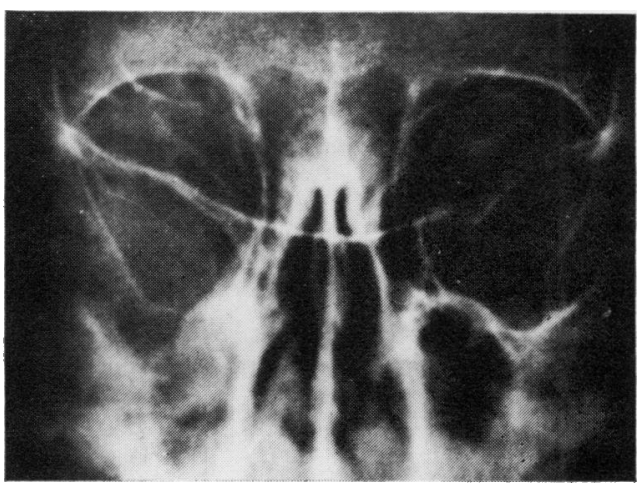

FIG. I I Case 2. Postero-anterior $x$ ray view of skull, showing right opaque maxillary antrum and destruction of orbital bony floor by a reticulum cell sarcoma of the maxillary sinus 
count showed 7 per cent. monocytes. Orbital exploration revealed the extension of a maxillary sinus tumour to the orbit. Histopathological examination of a biopsy of the tumour showed a reticulum cell sarcoma (Fig. 12). Radiotherapy was continued by $x$ ray, but 3 years later the patient died of cachexia.

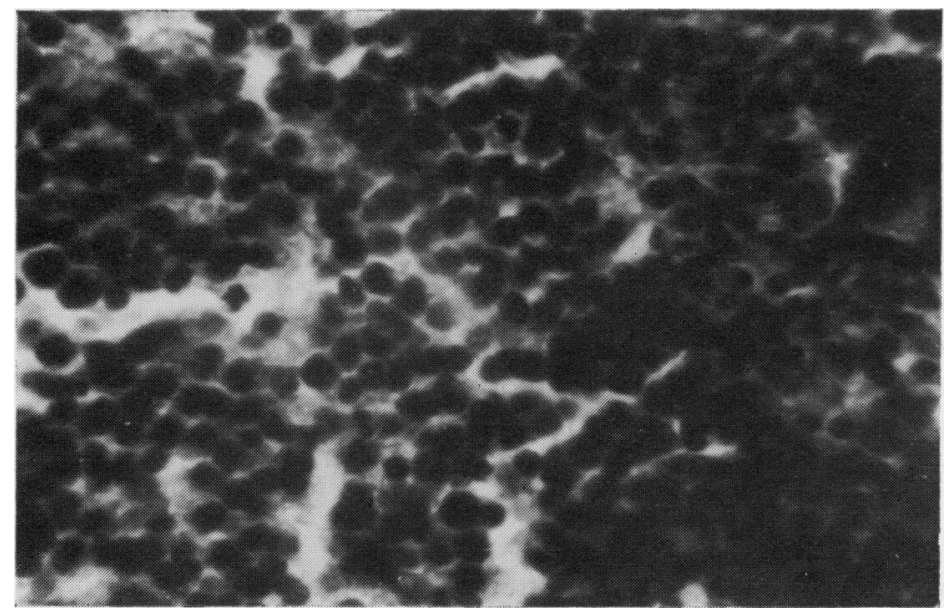

FIG. 12 Case 2. Section of reticulum cell sarcoma of maxillary sinus invading the orbit. $\times 54^{\circ}$

\section{Summary}

Two very rare cases are reported in which a recurrence of proptosis was due to a second tumour of different pathology.

In the first case the proptosis was originally due to a benign encapsulated haemangioendothelioma and in the second to a cavernous haemangioma. Some years after successful surgical removal of these benign orbital tumours, proptosis recurred in the same eye. The cause in each patient was a malignant tumour invading the orbit from the maxillary sinus; one was a carcinoma and the other a reticulum cell sarcoma. 